\section{Stress-Induced Thermal and Shrinkage Strains in CONCRETE}

By Zdeněk P. Bažant,' F. ASCE, and Jenn-Chuan Chern²

Abstanct: A previous material model for the increase of creep of concrete caused by simultaneous drying is extended to describe the effect of both drying and wetting, as well as the increase of creep caused by temperature changes, both heating and cooling. By theoretical arguments and comparisons with mumerous existing arown that the comparisons with shown that the creep increase due to temperature changes, sometimes called the transitional thermal creep, is physically the same phenomenon as the increase of creep due to humidity changes, known as the Pickett effect (or the drying creep effect). In accord with the previous model for drying creep alone, the present extended model explains the increase of creep due to humidity or temperature changes as a consequence of principally two effects: (1) Stress-induced shrinkage (or swelling) or stress-induced thermal expansion (or contraction); and (2) the distributed tensile cracking (or strain-softening) of concrete. The former effect is explained by the previously advanced hypothesis that the creep viscosity depends on the pore humidity rate. The latter effect reduces measured overall deformations, and thus results in the true thermal expansion or shrinkage of uncracked material being considerably larger than observed on load-free companion specimens when significant nonuniformly distributed self-equilibrated stresses are produced by the temperature or humidity change. The proposed material model is suitable for finite-element programs.

\section{INTRODUCTION}

A change in temperature of concrete during its creep under load causes a significant transient increase of creep (Fig. 1). This effect, which has been sometimes referred to as the transitional thermal creep $(14,16,18,24,28)$, occurs for both heating and cooling. It has been regarded as a special, peculiar property of concrete, which added further complexity to the multitude of various effects exhibited by this common yet intriguing material.

The reality, however, is simpler. In a previous work (10) it was suggested that what had been known as the transitional thermal creep is in fact the same phenomenon as the Pickett effect, also known as the drying creep effect. That this suggestion is indeed true will be demonstrated in this study by analysis of the test data that exist in the literature. For this purpose, the previous theory (10), which represents a special case of an earlier thermodynamic theory for the role of moisture migration in concrete creep $(3,4)$, will be extended by making it applicable not

'Prof. of Civ. Engrg., Northwestern Univ., Evanston, IL 60201.

${ }^{2}$ Res. Engr., Div. of Reactor Analysis and Safety, Argonne Natl. Lab., Argonne IL 60439; presently Assoc. Prof. of Civ. Engng., Taiwan Natl. Univ., Taipei, Repub. of China.

Note. Discussion open until March 1, 1988. To extend the closing date one month, a written request must be filed with the ASCE Manager of Journals. The manuscript for this paper was submitted for review and possible publication on July 28, 1986. This paper is part of the Journal of Engimeering Mechamics, Vol 113, No. 10, October, 1987. (C)ASCE, ISSN 0733-9399/87/0010-1493/\$01.00. Paper No. 21881.

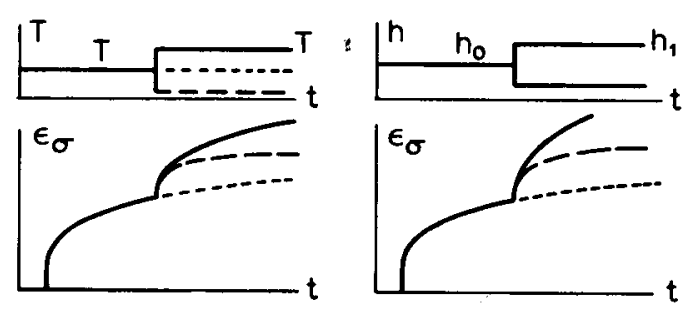

FIG. 1. Effect of Temperature or Humldity Change during Creep $\left(e_{\mathrm{v}}=\right.$ StressProduced Strain)

only for drying, but also for wetting and for temperature changes of either sign.

We will see that the creep increase due to humidity as well as temperature changes is a consequence of two phenomena: (1) The stressinduced shrinkage and stress-induced thermal expansion (termed swelling or contraction if negative), which arise from the effect of microdiffusion of water between capillary pores and gel pores on the rate of bond breakages that are the source of creep, and (2) the effect of tensile cracking (strain-softening), which causes the strain of a load-free companion specimen to be much less than the true material shrinkage or expansion and represents an apparent mechanism in Wittmann's terminology (31).

The existence of stress-induced thermal expansion was pointed out in a preceding paper (10) as a consequence of the fact that the chemical potential of pore water in concrete must depend both on pore relative humidity (relative vapor pressure) and temperature. A similar concept of stress-induced thermal expansion was recently independently proposed and experimentally justified by Thelandersson $(26,27)$, although without any relation or equivalence to the stress-induced shrinkage and without theoretical derivation on the basis of thermodynamics and a hypothesis about a microdiffusion mechanism. It may be also pointed out that the stress-induced shrinkage, as well as the stress-induced thermal expansion, represents a special limiting case of an earlier thermodynamic theory for the role of pore water in concrete creep $(3,4)$ when it is assumed that the microdiffusion of water between the capillary pores and the gel pores is infinitely fast (compared to the macroscopic diffusion of water through concrete specimens or structures). This assumption is probably very good although its verification by direct measurement seems impossible.

\section{Review of Model for Creep with Cracking at Variable Humidity}

To satisfy the condition that for a large tensile strain the maximum principal tensile stress must reduce to zero, it is necessary that the column matrix $\varepsilon$ of the strains due to cracking be additive to the column matrices for the elastic strains with creep $e$, for shrinkage or swelling $\boldsymbol{\varepsilon}_{s}$, and for thermal strain $\varepsilon_{t}$ (also called thermal dilatation or thermal expansion). Thus, the matrix of the total strain in concrete may be 


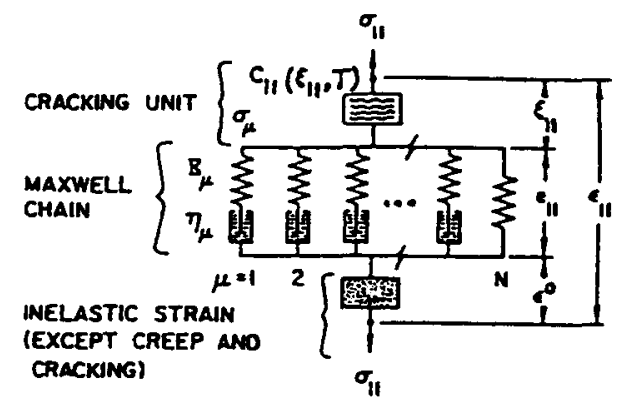

FIG. 2. Maxwell Chain Model for Linear Aging Creep with Elasticity, Coupled in Series with Cracking (Strain-Softening) Element and Shrinkage-Thermal Expansion Element (after Ref. 2)

expressed as

$\varepsilon=\mathbf{e}+\xi+\varepsilon_{s}+\varepsilon_{t}$

This equation may be illustrated by the rheologic model (Fig. 2) in which the system of springs and dashpots that describe creep with elastic deformation is coupled in parallel with a cracking element and an element that describes shrinkage (or swelling) and thermal strains. The progressive distributed cracking which is manifested by strain-softening is most easily described by a secant stress-strain relation

\section{$\sigma=\mathbf{C}(\xi, T, h) \xi$}

$\mathrm{C}$ is a $6 \times 6$ matrix of secant elastic moduli, which depends on the cracking strain and possibly also on temperature $T$ and pore relative humidity $h$. In the simplest possible formulation, all the elements of matrix $\mathbf{C}$ are zero except the first three diagonal ones, associated with the normal stresses and strains. These diagonal elements of matrix $\mathbf{C}$ may be approximately described as functions of the corresponding cracking strain, i.e., $\mathbf{C}=$ $\mathbf{C}_{\alpha \alpha}\left(\xi_{\alpha \alpha}\right)$ in which $\alpha=1,2,3$. A convenient expression for this function is $\mathbf{C}_{\alpha \alpha}=\mathbf{E}_{s} \exp \left(-c \xi_{\alpha \alpha}^{s}\right)$ in which $E_{s}, c$, and $s=$ material constants $(10,11)$. Repetition of Greek subscripts implies no summation.

The viscoelastic strain e may be effectively represented by the Maxwell chain model $(4,6)$, according to which the stress-strain relation for the viscoelastic deformations is

$\sigma=\sum_{\mu=1}^{N} \sigma_{\mu}$

$\dot{\mathbf{e}}=\frac{1}{E_{\mu}\left(t_{e}\right)} \mathbf{B} \dot{\sigma}_{\mu}+\frac{1}{\eta_{\mu}\left(t_{e}\right)} \mathbf{B}^{\prime} \boldsymbol{\sigma}_{\mu}$

$\boldsymbol{\sigma}_{\mu}=$ the column matrix of the partial strains (internal variables) associated with the $\mu$ th unit of the Maxwell chain $(\mu=1, \ldots N)$, and $E_{\mu}$ and $\eta_{\mu}=$ the elastic moduli and viscosities of the $\mu$ th Maxwell unit. These parameters are functions of the equivalent hydration period $t_{e}$ (or maturity). B and $B^{\prime}$ are $6 \times 6$ constant matrices that express the conditions of material isotropy and are defined in terms of Poisson ratio. These matrices are symmetric and $B_{11}=B_{22}=B_{33}=1 ; B_{44}=B_{55}=B_{66}=1+\nu$; and $B_{12}=$ $B_{23}=B_{13}=-v$, all other matrix elements being zero. The moduli $E_{\mu}$ as functions of $t_{e}$ may be obtained from a given compliance function of concrete creep by a well-known efficient algorithm $(4,6,22)$.

The viscosity coefficients and the equivalent hydration period may be further expressed as (10)

$$
\begin{gathered}
\frac{1}{\eta_{\mu}\left(t_{e}\right)}=\frac{\phi_{T} \phi_{h}}{\tau_{\mu} E_{\mu}\left(t_{e}\right)} \\
t_{e}=\int \beta_{T} \beta_{h} d t \ldots
\end{gathered}
$$

in which $\tau_{\mu}$ are properly chosen relaxation times $(4,6,22) ; t=$ time; $\phi_{T}$ and $\beta_{T}=$ functions of temperature $T$; and $\phi_{h}$ and $\beta_{h}=$ functions of pore relative humidity $h$. The temperature effect may be given according to the activation energy concept, in which case $\phi_{T}=\exp \left[\left(T_{0}^{-1}-T^{-1}\right) U_{a} / R\right]$; and $\beta_{T}=\exp \left[\left(T_{0}^{-1}-T^{-1}\right) U_{h} / R\right]$ where $T_{0}=$ reference temperature $\left(25^{\circ} \mathrm{C}\right) ; R=$ universal gas constant; and $U_{a}$ and $U_{h}=$ the activation energies for the creep rate and for the hydration rate. The influence of humidity may be approximately characterized by the empirical expressions $\phi_{h}=\alpha_{h}+(1-$ $\left.\alpha_{h}\right) h^{2}$; and $\beta_{h}=\left[1+(a-a h)^{4}\right]^{-1}$, in which $\alpha_{h}$ and $a$ are constants $(a \simeq 5)$. These expressions were justified by analysis of test data in Bažant (4).

When the normal stresses exceed approximately $40 \%$ of the strength, one should take into account the nonlinear dependence on stress. As described in Ref. 10, this nonlinearity may be most simply characterized by replacing in the stress-strain relation the values $e_{11}, e_{22}$, and $e_{33}$ by $e_{11} f_{1}, e_{22} f_{1}$, and $e_{33} f_{1}$ in which $f_{1}=$ a function of the normal stress.

The shrinkage or swelling at zero stress may be approximately described by the relations (10):

$\dot{\varepsilon}_{s}=\kappa h$

$\kappa=\varepsilon_{s}^{0} \psi$

$\psi=g_{s}\left(t_{e}\right) \frac{d f_{s}(h)}{d h}$

in which $\kappa=$ shrinkage coefficient $; \varepsilon_{s}^{0}=$ constant; $g_{s}\left(t_{e}\right)=$ a given function of age $t_{e}$ which characterizes the reduction in shrinkage due to the hardening caused by continuing hydration; and $f_{s}(h)=$ function that defines shrinkage at zero stress as humidity $h$ is varied. Approximately, $f_{s}(h)=1-h^{3}$.

\section{Effect of Variable Temperature and Humidity}

One mechanism that causes an increase of the creep rate during drying is the microcracking or strain-softening produced by shrinkage stresses (Fig. 3). As a result, the true material shrinkage is considerably higher than that measured on a shrinkage specimen, and thus the creep strain obtained by subtracting the deformation of a companion shrinkage specimen from 


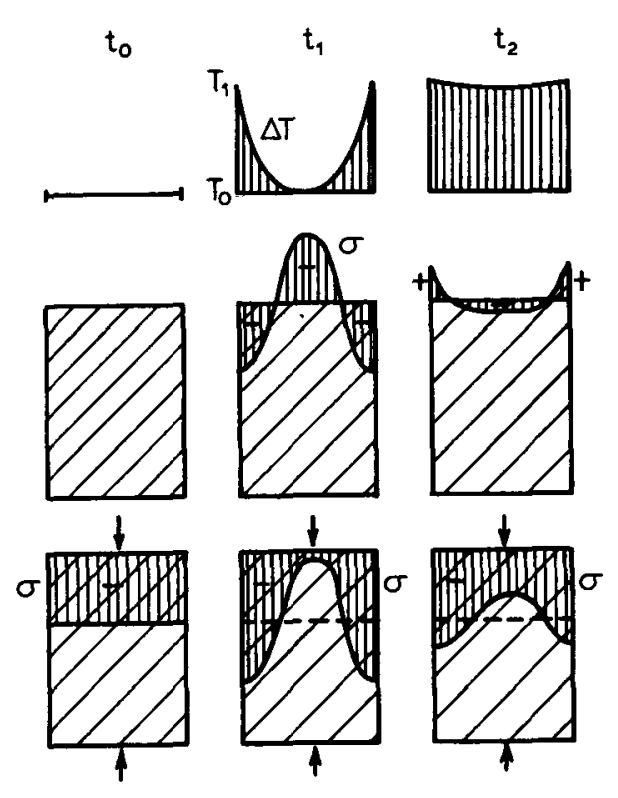

FIG. 3. Stresses and Cracking Induced In Layers of Loaded or Load-Free Speclmens at Change of Temperature

the deformation of a loaded specimen is considerably larger than it would be if the true material shrinkage were subtracted. The role of strainsoftening or cracking was analyzed in the early 1970's by Bažant and Wu (12), and later Wittmann and Roelfstra proposed that cracking, as an apparent mechanism, can explain essentially all of the drying creep phenomenon. However, detailed finite-element analyses have revealed that this is not the case (10). To be sure, Wittmann and Roelfstra (32) were right when they identified cracking as a major contribution to the drying creep effect. However, the difference between the true material shrinkage and the apparent shrinkage observed on a companion specimen could explain the increase of creep at drying only if the creep viscosities $\eta_{\mu}$ were independent of the pore humidity $h$. In reality, as discovered during the 1960 's, $(13,23,30,31)$, the creep viscosities strongly increase as $h$ decreases, i.e., the lower the moisture content the smaller is the creep rate, provided that the moisture content is constant in time (i.e., the pore water is in thermodynamic equilibrium). If the decrease of creep rate due to a lowered pore humidity is taken into account, the deformation difference between a loaded drying specimen and a load-free drying companion specimen is not large enough to yield an increase of deformation compared to the creep of a loaded, sealed specimen. At most, it can merely offset the reduction of creep due to a lowered moisture content.

This conclusion, which resulted from extensive finite-element analyses of numerous test data from the literature, requires that there exists an additional physical mechanism on the microstructural level that causes an increase in the long-time deformations during drying. Such a mechanism is probably the effect of microdiffusion of water in cement gel on the rate of bond breakages in the solid microstructure which are imagined to be the source of creep $(3,4,6,10)$.

As shown by thermodynamic arguments in a previous work (10), this physical mechanism, which is a special simplified case of that proposed about 15 years ago $(3,4)$, is described by the hypothesis that creep viscosity $\eta$ is a function of the microdiffusion flux of water $j$, i.e., $\eta=\eta(j)$ (for the moment, we now drop subscript $\mu$ from $\eta_{\mu}$ and consider a single Maxwell unit instead of the Maxwell chain). The hardened Portland cement paste is a double porosity material, whose pore space includes capillary pores (the macropores) as well as far smaller gel pores (the micropores, usually considered under $30 \AA$ thick). The macroscopic diffusion of water through concrete passes predominantly through the macropores, although the passages must include some micropore-sized necks between adjacent capillary pores since the capillaries (in a good quality concrete) are not continuous. A microdiffusion in cement gel, consisting of a local flow of water between the gel pores and the adjacent capillary pores, can be induced only by a change in the chemical potential (Gibbs free energy/unit mass) of water in the capillary pore. This potential is a function of the pore relative humidity $h$ and temperature $T$. Thus, an imbalance in chemical potential between the molecules of water in the capillary pore and the adjacent gel pores is produced by a change in pore humidity as well as temperature. This then produces a local flow of water molecules into the gel pores or out of them. It is hypothesized that the flow through pores of molecular dimensions in the cement gel promotes the bond breakages that cause creep.

The microdiffusion of water between the capillary pore and the adjacent gel pores must happen, on the average, equally in all directions, and so the flux $j$ must be a scalar. From the viewpoint of a macroscopic smoothing continuum, this flow happens at a point, i.e., $j$ is a point property. Mathematically, our basic hypothesis is that $\eta($ or $\eta \mu)$ is a function of $j$. Now, as a result of the observation that the diffusion half-time for the microdiffusion must be far shorter than that for the macrodiffusion through the entire specimen, as introduced in Ref. 10 , flux $j$ is a function of the time rate $\dot{\mu}$ of the chemical potential of water in the macropores. At the same time, this chemical potential is a function of $h$ and $T$, which may be approximately considered as linear for our purposes. This leads to the conclusion that flux $j$ may be described as $j=a_{1} \dot{H}$ where (10)

$H=h+a_{T} t \quad\left(a_{T}>0\right)$

in which $a_{T}=$ a coefficient that is independent of $\dot{H}$ and $\dot{T}$. Superimposed dots denote the time rates.

In the preceding work (10), it was assumed that creep fluidity (i.e., inverse viscosity) $1 / \eta$ was a linear function of $j$. Based on subsequent experience with the fitting of further test data, it now appears to be more appropriate to consider $1 / \eta$ as a function of $|j|$ rather than $j$. This means that the breakages of bonds are assumed to be promoted by both positive $j$ (flux into the gel pores) and negative $j$ (flux out of the gel pores). In the previous work (10), this distinction was not necessary since only test data for drying, for which $j>0$ and $h<0$, were considered. So we introduce the hypothesis that 
$\frac{1}{\eta}=\frac{1}{\eta}-\kappa^{\prime}|\hat{H}|$

in which the basic viscosity $\eta$ and coefficient $\kappa^{\prime}$ are independent of $\dot{h}$ and $\dot{T}$. Now, noting that $|\dot{H}|=\dot{H}$ sign $\dot{H}$ and substituting Eq. 7 into the equation for a single Maxwell unit $(\dot{\sigma} / E)+(\sigma / \eta)=\dot{\varepsilon}-\bar{k} h-\bar{\alpha} \dot{T}$ we obtain

$\frac{\dot{\sigma}}{E}+\sigma\left[\frac{1}{\eta}-\kappa^{\prime}\left(h+a_{T} T\right) \operatorname{sign} H\right]=\dot{\varepsilon}-\bar{\kappa} h-\bar{\alpha} T$

The bracketed term represents $1 / \eta$; and $\bar{\kappa}$ and $\bar{\alpha}=$ the stress-independent shrinkage coefficient and thermal expansion coefficient, respectively; sign $\dot{H}=1$ if $\dot{H} \geq 0$, and sign $\dot{H}=-1$ if $\dot{H}<0$. Combining algebraically the terms with $\dot{h}$ and $\dot{T}$, Eq. 8 can be rewritten as

$\frac{\dot{\sigma}}{E}+\frac{\sigma}{\eta} \doteq \dot{\varepsilon}-\kappa h-\alpha \dot{T}$

in which

$\kappa=\varepsilon_{s}^{0} \psi(1-r \sigma \operatorname{sign} H)$

$\alpha=\bar{\alpha}(1-p \sigma \operatorname{sign} \dot{H})$

with the notation $r=\kappa^{\prime} / \bar{k}$, and $\rho=\alpha^{\prime} / \bar{\alpha}$. The basic thermal expansion coefficient $\bar{\alpha}$ is independent of stress as well as $\dot{h}$ and $\dot{T}$, but it can (and probably does) depend on pore humidity $h$.

In the preceding paper (10), the expressions $\kappa=\varepsilon_{s}^{0} \psi(1+r \sigma)$; and $\alpha=$ $\bar{\alpha}(1+\rho \sigma)$ were used. Nevertheless, the data fitting in that work is compatible with the present formulation because only the case of drying, for which sign $\dot{H}$ is negative, and constant temperature were considered. The fact that Eq. 7 should involve $|\dot{H}|$ rather than $\dot{H}$ and that the stress in Eqs. 10,11,13, and 14 should be multiplied by sign $\dot{H}$ was recently proposed by Bažant (7) on the basis of the theoretical argument just sketched.

The advantage of including the effect of microdiffusion flux $j$ in the shrinkage and thermal expansion terms (Eqs. 9-11) rather than in the viscosity term (Eq. 8) is the possibility of a simpler generalization to the Maxwell chain, which is required when creep over a broader time range is considered. If the terms with $\kappa^{\prime}$ were retained in the viscosity term as in Eq. 8, it would be necessary to include such terms for each of the viscosities $\eta_{\mu}$, in general with different coefficients for each unit of the chain. On the other hand, when these terms are included on the right-hand side of Eq. 9, generalization for Maxwell chain is easy and requires no additional parameters; it suffices to replace the left-hand side of Eq. 9 with $\dot{\sigma}_{\mu} / E_{\mu}+\sigma_{\mu} / \eta_{\mu}$

Further generalization to three dimensions may be written, in view of Eq. 1, as follows:

$\dot{\mathbf{e}}=\dot{\boldsymbol{\varepsilon}}-\mathbf{k} \boldsymbol{h}-\alpha \dot{T}-\dot{\xi}$

in which $\dot{\mathrm{e}}=$ the column matrix of creep plus elastic strains; $\dot{\mathrm{e}}$ is expressed on the basis of the Maxwell chain model according to Eq. 3; and $\kappa$ and $\alpha$ $=$ the column matrices of the shrinkage coefficients and the thermal expansion coefficients. The elements of these $6 \times 1$ column matrices consist of the following generalizations of Eq. 10 and 11 :

$\kappa_{i j}=\varepsilon_{s}^{0} \psi\left[\delta_{i j}-\left(r \sigma_{i j}+r^{\prime} \sigma_{0} \delta_{i j}\right) \operatorname{sign} \dot{H}\right]$

$\alpha_{i j}=\bar{\alpha}\left[\delta_{i j}-\left(\rho \sigma_{i j}+\rho^{\prime} \sigma_{0} \delta_{i j}\right) \operatorname{sign} \dot{H}\right]$

in which $\sigma_{0}=\sigma_{k k} / 3=$ mean stress; $\delta_{i j}=$ Kronecker delta; and subscripts $i, j$ in Eqs. 13 and 14 refer to Cartesian coordinates $x_{i}, i=1,2,3$. Eqs. 13 and 14 are the most general possible generalizations of Eqs. 10 and 11 , such that the condition of material isotropy remain satisfied. However, for the same reasons as mentioned before $(10)$, it seems that $r^{\prime} \simeq \rho^{\prime} \simeq 0$. From experience it appears that usually the values of $r$ and $\rho$ are between 1 and $6 \mathrm{psi}^{-1}$.

The constitutive relation is now completely specified by Eqs. 2, 3, 6, 12, 13, and 14. A finite-element program based on this constitutive relation has been written and used to analyze various test specimens. Integration in time is carried out in time steps, using quasielastic incremental formulas which were presented previously (11). These formulas make it possible to increase the time step to very long intervals if the rates of strains and stresses decay with time. The finite-element program solves also in each time step the pore humidity distribution throughout the finite-element system, based on specified boundary conditions for humidity. In this calculation, the dependence of moisture diffusivity $C$ on the equivalent hydration period $t_{e}$, as well as on pore humidity $h$, is easily taken into account, as described before (10). However, a possible effect of cracking on the effect of diffusivity of moisture is neglected for lack of test data.

For the purpose of the present study, the program developed before $(10,11)$ has been extended to variable temperature. The heat conduction equation is solved by finite elements to yield the temperature distribution at each time step, along with the value of $\dot{T}$ in each finite element.

The principal result of the present theoretical analysis is the dependence of the shrinkage and thermal expansion coefficients on stress $\sigma$, as given by Eqs. 10 and 11. The stress-dependent terms in these equations may be called the stress-induced shrinkage and the stress-induced thermal expansion. If the present theoretical argument is correct, then the existence of stress-induced shrinkage must imply the existence of stress-induced thermal expansion, and vice versa. This is because the value of the chemical potential of pore water depends both on pressure and temperature.

The concept of stress-induced thermal expansion was independently proposed on the basis of experimental data (1) by Thelandersson $(26,27)$. Both the phenomena of stress-induced thermal expansion and stressinduced shrinkage were also implied indirectly in the formulation of Ref. 3 (see Eq. 21 of Ref. 10). It is the intent of the present study to show that the stress-induced thermal expansion is inseparable from the stress-induced shrinkage and represents one and the same physical phenomenon. This was in general terms already suggested in the previous study (10).

The increase in the creep rate at a rapid change of temperature, discovered experimentally during the 1970's (e.g., Ref. 5), was originally called the transitional thermal creep and has been considered as an additional new phenomenon. In this study it is argued that this phenomenon is in fact the same as the stress-induced shrinkage, i.e., as the drying 
creep or the Pickett effect. No new mathematical formulation and no further material constants need to be introduced to model the so-called transitional thermal creep. In this sense we find that the reality is simpler than previously thought - a gratifying outcome for a theoretical investigation

\section{Analysis of Test Data}

The finite element program used in data fitting was based on the assumption of plane cross sections remaining plane. For beam specimens, the cross section was subdivided into square elements; see Ref. 10. For all other specimens, an axisymmetric finite-element program was used.

The finite-element program has been used to analyze the basic test data available in the literature in order to calibrate the unknown material parameters and determine whether the data can be fitted in a consistent manner. Difficult though this task might seem, it is nevertheless quite feasible since all the material parameters need not be determined simultaneously but can be identified sequentially. From the basic creep data, consisting of the values of the compliance function $J\left(t, t^{\prime}\right)$, one can determine $\tau_{\mu}$ and $E_{\mu}\left(t_{e}\right)$ by a well-known algorithm (6). If the basic creep data are missing, $J\left(t, t^{\prime}\right)$ is predicted according to the BP model. If the data are incomplete, the most frequent case, the prediction of the BP model is adjusted to fit the available data. Coefficients $\phi_{t}, \phi_{h}, \beta_{t}$, and $\beta_{h}$ can be predicted from the formulas that are mentioned in connection with Eq. 4 and were developed before. Function $g_{s}\left(t_{e}\right)$ (Eq. 5) results from shrinkage tests for various ages of concrete at the start of drying or at various specimen sizes. Function $f_{s}(h)$ (Eq. 5$)$ is determined primarily by shrinkage tests at various environmental humidities. Coefficient $f_{1}$ for stress nonlinearity in compression has only minor importance for fitting the present type of data and can be taken according to the uniaxial compression stress-strain diagram (Eq. 4 in Ref. 10). The dependence of moisture diffusivity $C$ on $t_{e}$ and $h$ can be taken as determined before (10) from drying tests. Approximate values of the thermal diffusivity, if not reported, can also be taken from the literature. The function $C_{11}\left(\xi_{11}, T\right)$, which characterizes strain-softening due to microcracking, may be identified from stabilized uniaxial tensile tests, as done before (11). This leaves only two coefficients $\varepsilon_{s}^{0}$ and $r$ to be determined from the deformation of loaded and load-free specimens caused by a change of environmental humidity at constant temperature, and only three coefficients, $\bar{\alpha}, \rho$ and $a_{T}$, to be determined from deformation measurements on load-free and loaded specimens at variable temperature and, preferably, at constant moisture content. The values of the material parameters corresponding to the individual fits shown in various figures are given either in the figure or in Appendix I.

The fact that Eqs. 13 and 14 should indeed involve sign $H$ is corroborated by comparing the effects of heating and cooling as well as the effects of drying and wetting. Since both of these generally tend to increase the deformation, $|H|$ instead of $\dot{H}$ is needed in Eq. 7, and sign $\dot{H}$ in Eqs. 10-14.

The primary motivation for the present study was the modeling of concrete response at high temperatures. The strain-softening due to microcracking at high temperatures was checked against the post-peak
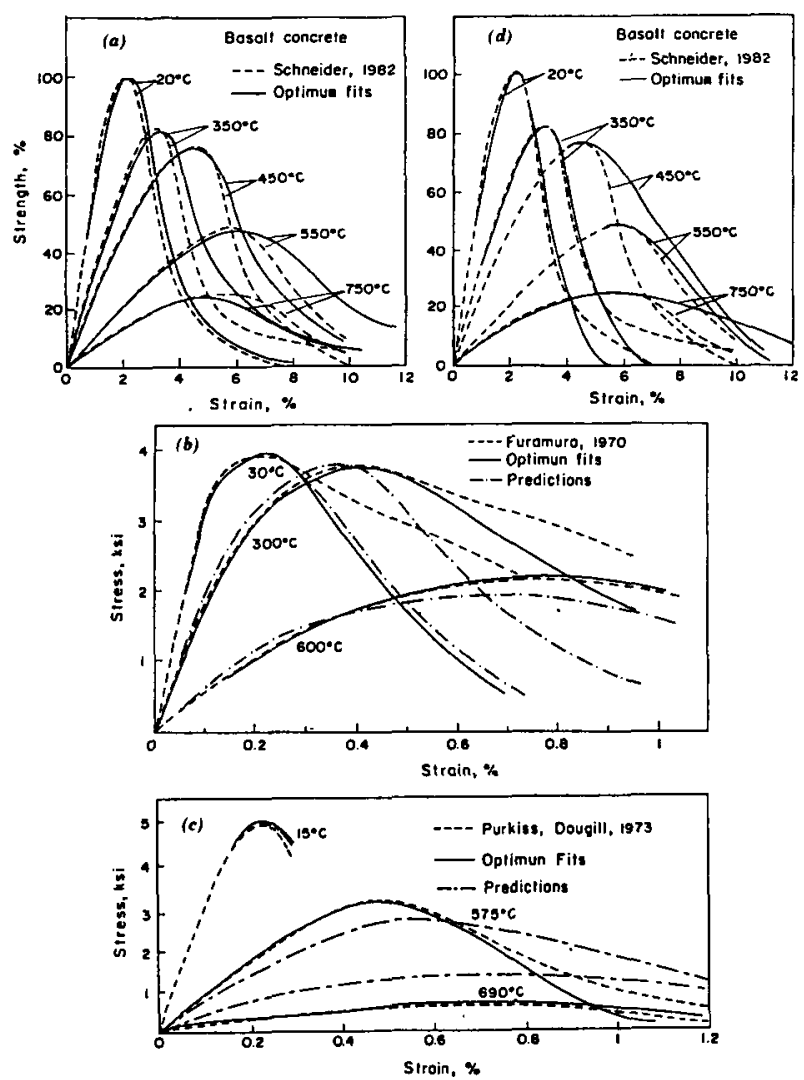

FIG. 4. (a) Schnelder's (18) Tests at Constant Stress Rate and Various Temperatures; (b) Compression Stress-Strain Curves Measured by Furamura (19); (c) Compression Stress-Straln Curves Measured by Purkiss (20); (c) Predictions of Concrete Response for Schneider's Tests

compression stress-strain curves measured by Schneider (25), Furamura (15), and Purkiss (21); see Fig. 4(a-c). Despite a significant stress drop due chiefly to strain-softening (though partly also to stress relaxation), there were no visible macrocracks in the specimens, i.e., strain softening was due to invisible microcracking. Fig. 4 also shows the fits of these data with the present model, taking into account, of course, the increased creep at high temperatures.

Fig. 5 shows test data for the effect of temperature on the elastic Young's modulus, the compressive strength, and the tensile strength. The data were directly digitized at small temperature intervals, and linear interpolation within these intervals was then used in the computer program. The stress-strain curves calculated in this manner are shown in Fig. $4(b-d)$. The temperature contribution to these curves was calibrated from the data shown later in Fig. $5(a)$. Subtracting this contribution one can 

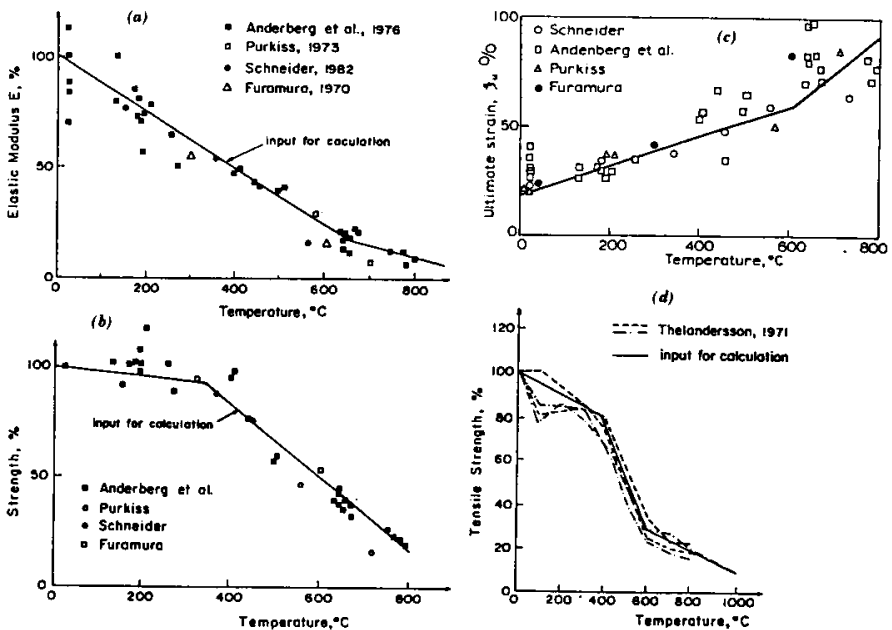

FIG. 5. Effect of Temperature on: (a) Statlc Elastic Modulus; (b) Compressive Strength; (c) Ultimate Strain, Measured on Heated Unstressed Specimens; (d) Split Cylinder Tensile Strength in Hot State and after Cooling (Normal Concrete)

obtain the remaining strains, which have to be described by the strainsoftening element.

Fig. 6 shows Thelandersson's measurements of the restraining force caused by heating at zero strain. The calculated curve agrees reasonably well with these data.

Fig. 7 $(a$ and $b)$ shows the importance of stress-induced thermal expansion. On the left side, the test data by Anderberg (1) are fitted without the stress-induced component (i.e., with $\rho=r=0$ ), and on the right side with this component. The difference between the results of the calculation is very large, and while there is a large discrepancy on the left side of Fig. 7 , a good agreement is seen on the right side. Fig. 8(a) extends this analysis to higher temperatures. Good fits were found to be impossible, but they become possible when the stress-induced components are included, as shown in Fig. 8(a). In both cases, the load history and the temperature history are the same. The load is applied before heating, while for curve a, the load is applied after the start of heating. We see that if the temperature rises while the specimen is under sustained load, the thermal expansion is substantially reduced.

Fig. 9 compares the calculations according to the present model with the test data obtained by Hollington (17) for the deflection history of beams under load, subjected at the same time to temperature cycles. Calculations show that moisture migration has a relatively small effect on the result, which is partly due to the large size of cross section, relatively high environmental humidity $(85-100 \%)$, and relatively low temperatures $\left(5-20^{\circ} \mathrm{C}\right)$. The beams are subjected to a uniform load of $56 \mathrm{lb} . / \mathrm{ft} .^{2}$ plus their own weight. While the mean prediction of the present theory is quite good, the fluctuating part of deflection is nevertheless underestimated.

Next we consider test data on transitional thermal creep $(14,16,28)$. Fig.

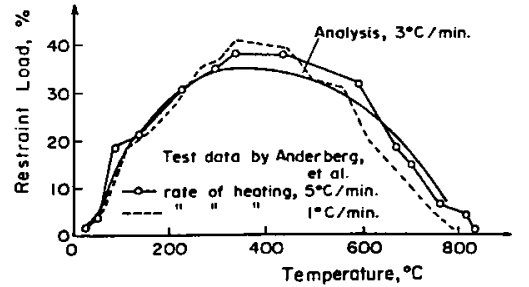

FIG. 6. Measured (15) and Calculated Axial Force (in Percent of Ultimate Load at Amblent Conditions) as Function of Temperature for Specimens Heated while Fully Restrained against Expansion; Heating Rate: (a) $5^{\circ} \mathrm{C} / \mathrm{min}$; (b) $1^{\circ} \mathrm{C} / \mathrm{min}$

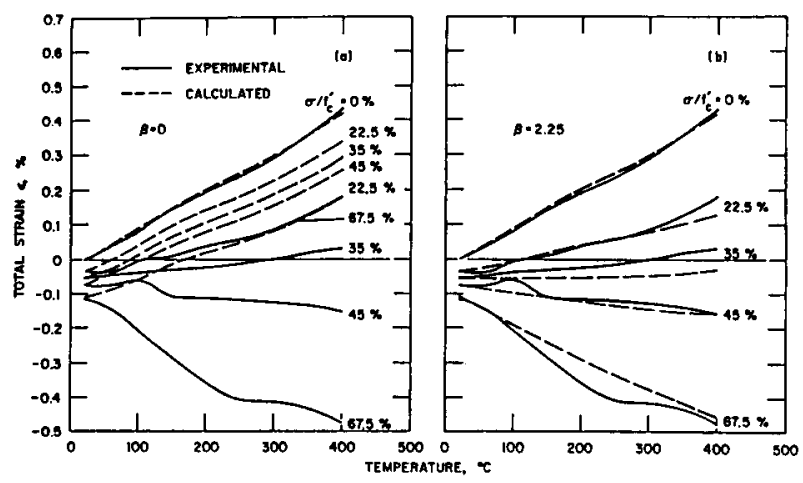

FIG. 7. Total Strain Response: (a) without Stress Dependence; (b) with Stress Dependence (after Ref. 16)

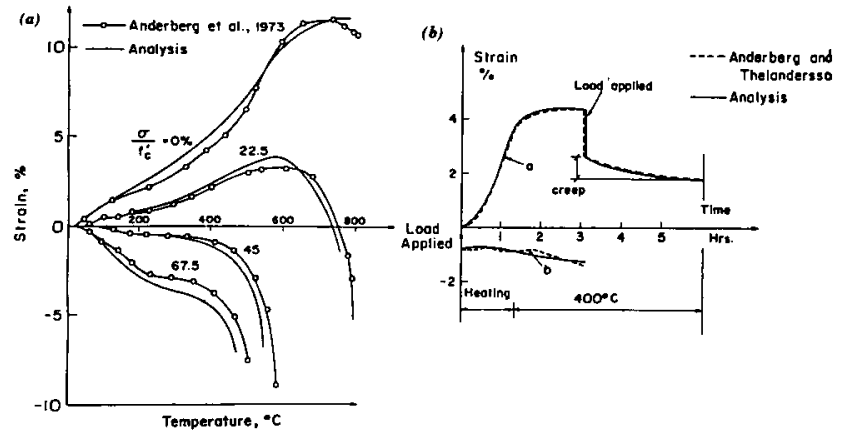

FIG. 8. (a) Deformations upon Heating (at $5^{\circ} \mathrm{C} / \mathrm{min}$ ) under Different Load Levels; (b) Measured Total Strain vs. Time for Isothermal Condition (Curve a) Compared with Strain Obtained If Load is Applied from Beginning (Curve b) (Temperature History Is Same in Both Cases) 

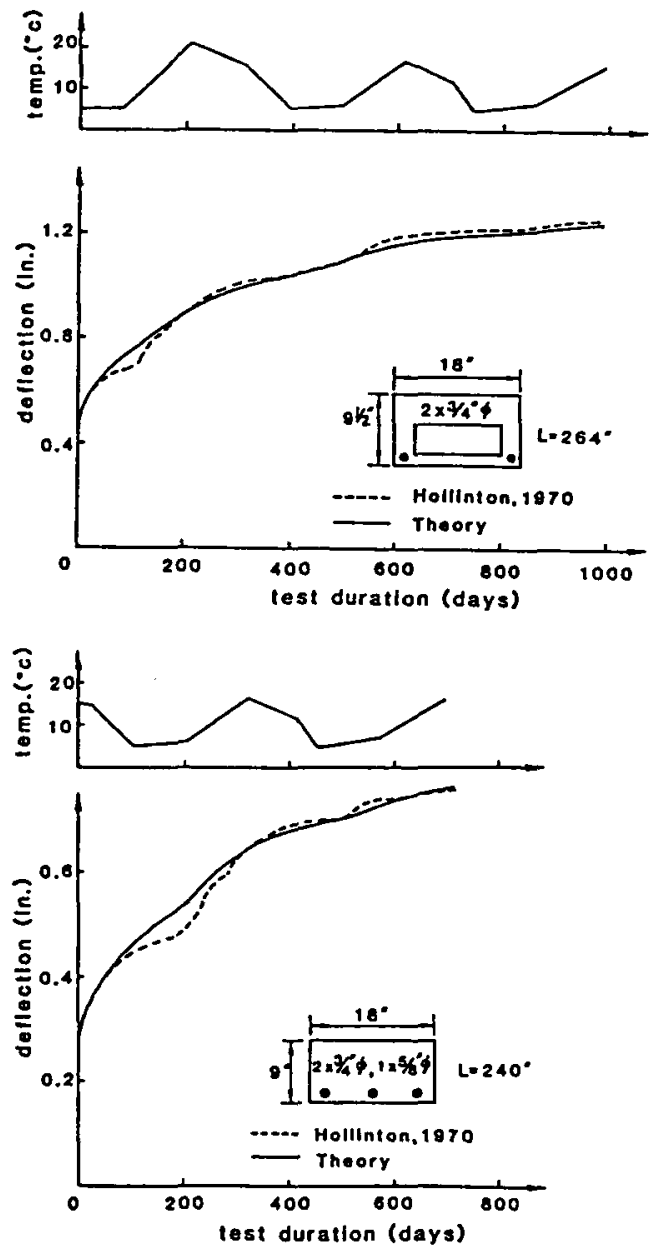

\section{FIG. 9. Test Data on Concrete Beam Deflection at Variable Temperature Compared} with Theory

10 shows Hanson's test data on the deflections of mortar beams of dimensions $0.8 \times 2 \times 10 \mathrm{in}$. which are immersed in water and are subjected to load before or after heating and to different heating rates. The apparent increase of the midspan deflection due to the transient thermal effect at an early stage of loading is seen to be quite significant. Furthermore, the beam that is heated faster deffects more. The present theoretical model fits the data well. The moisture effect can be neglected in the fitting of these data.

Fig. 11 shows the test results by Wallo, et al. (28) obtained for flexural loading of U-shaped specimens, with arms of cross section $1 \times 1$ in. and length 9.375 in. Fig. 11 shows that any change of temperature, either heating or cooling, temporarily accelerates the deflection increase. It is also interesting that the cooling process yields more deflection, but this is probably caused by the fact that the cooled specimen initially begins at high temperature at which the creep rate is also increased due to high age. The curves for the present theory agree with the data reasonably well.

Fig. 12 shows the test results of Fahmi and Bresler (14) obtained on hollow microconcrete cylinders with inner and outer diameters 5 and 6 in., loaded axially in compression. Due to the small thickness of the wall, the temperature quickly reaches a uniform distribution throughout the wall thickness, and so the transient temperature effect occurs for only a very short time period and is therefore rather small. However, the drying that is induced by heating is a much slower process, and its transient effect is in these tests the major cause of the increase in deformation, i.e., we see in these tests primarily the Pickett effect (stress-induced shrinkage or drying creep). For the tests at $50 \%$ environmental humidity, the Pickett effect is relatively short-lived, and later the deformation rate becomes very small due to low moisture content (Fig. 12). For comparison, the calculation results for creep at steady environmental conditions are also indicated in Fig. 12. We see that compared to this curve the transient effect is very significant.

Fig. 13 shows Schneider's test data for fast heating at high temperature $\left(300^{\circ} \mathrm{C}\right)$. The moisture and heat transfer at high temperature (over $100^{\circ} \mathrm{C}$ ) was calculated by the finite-element program TEMPOR2 based on the theory formulated in Ref. 5, 8, and 9. The specimens were cylinders of

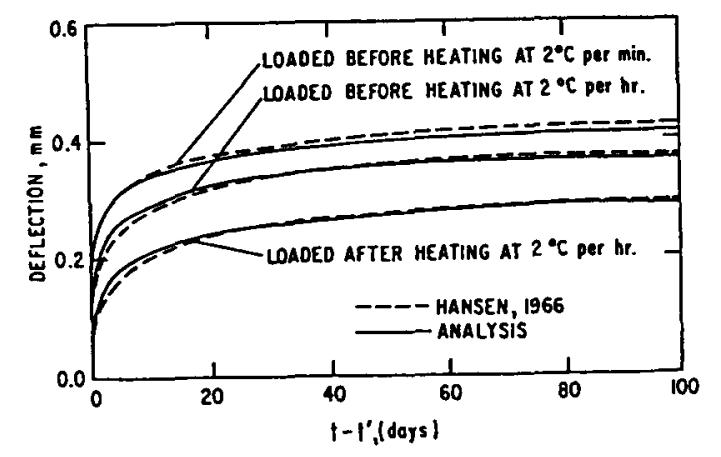

FIG. 10. Fits of Test Data by Hansen (22)

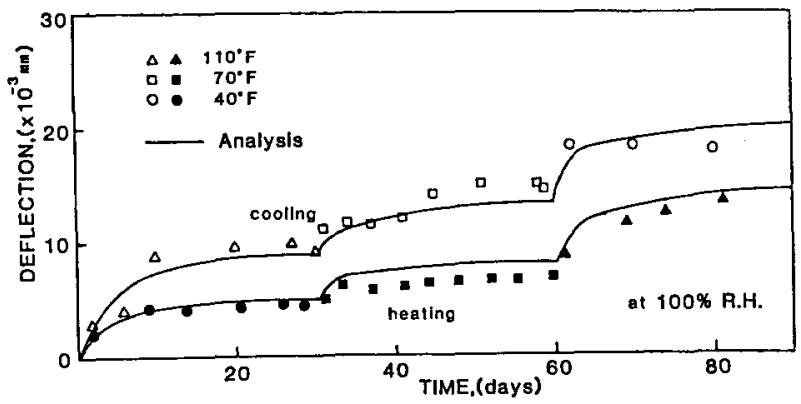

FIG. 11. Fits of Test Data by Wallo, et al. (23) 

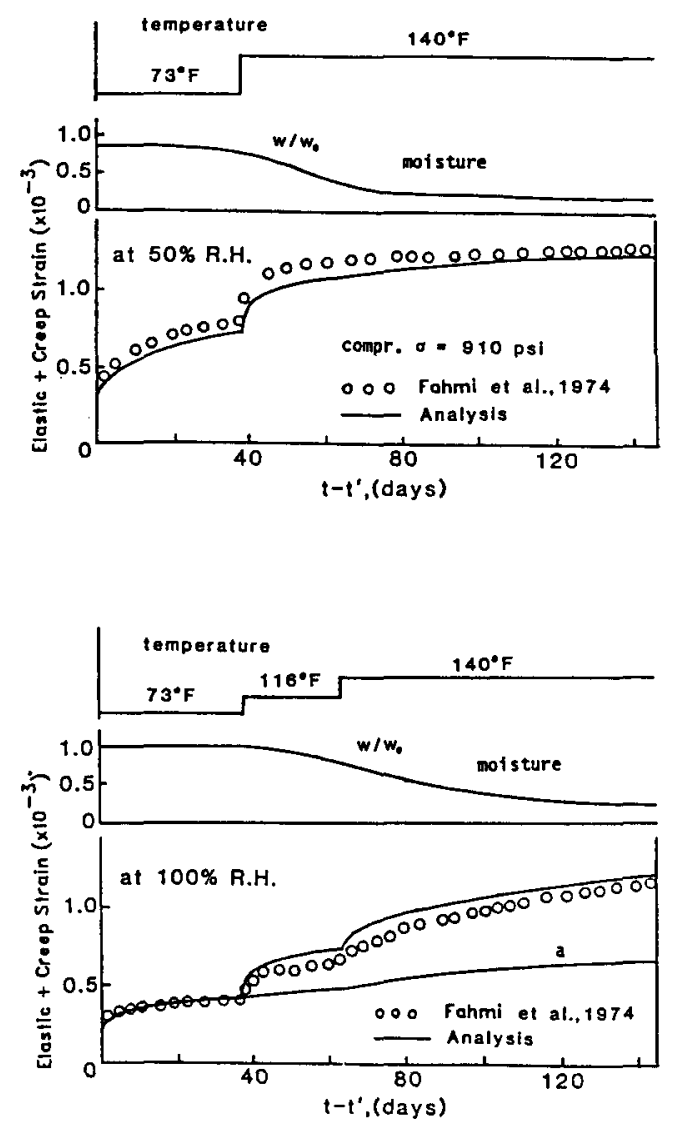

FIG. 12. Fits of Test Data by Fahmi, et al. (24)

diameter $3.15 \mathrm{in}$. and length $12 \mathrm{in}$. The rapid heating produces a rapid moisture loss from the surface layer of the specimen, while at the same time it drives part of the moisture toward the core at the beginning of heating, thus causing temporary water migration. In this type of test, the effect of $T$ on the deformation increase dominates, and the effect of $\dot{h}$ is much smaller.

Fig. 14 shows the original test results of Pickett (19), which first clearly documented the drying creep effect (Pickett effect). Plain concrete beams of $2 \times 2$ in. and span 32 in. were subjected to drying and wetting cycles. In addition to its own weight, the beam was loaded by a $50-\mathrm{lb}$. force at midspan. Curve a was obtained for loaded beams at cyclic humidity, curve b-loaded and drying, curve c-loaded but not drying, and curve ddrying but not loaded. The temperature was constant in these tests. We see that both drying and wetting increase the deflection and that the present theory can model these additional deflections due to humidity changes quite well. To model the deflection increase due to wetting, $\eta$ must be considered to depend on $|\dot{h}|$ rather than $\dot{h}$ or the stress-induced shrinkage

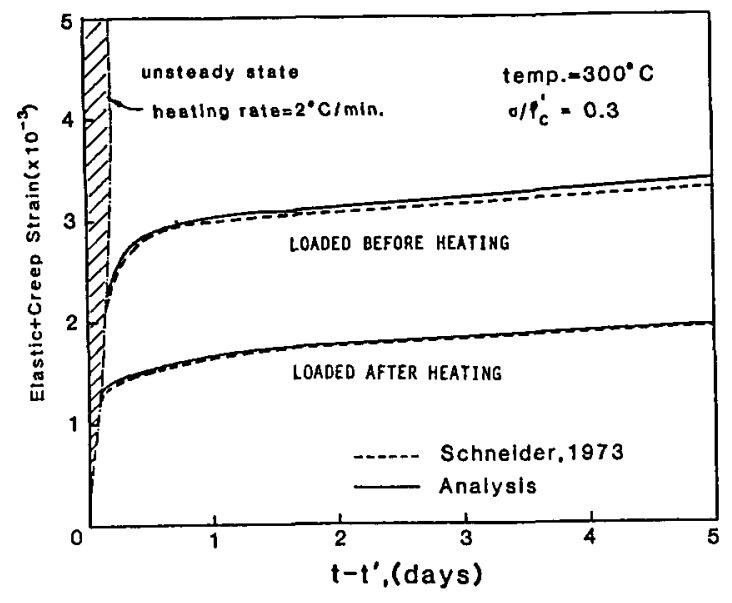

FIG. 13. Fits of Test Data by Schneider (25)

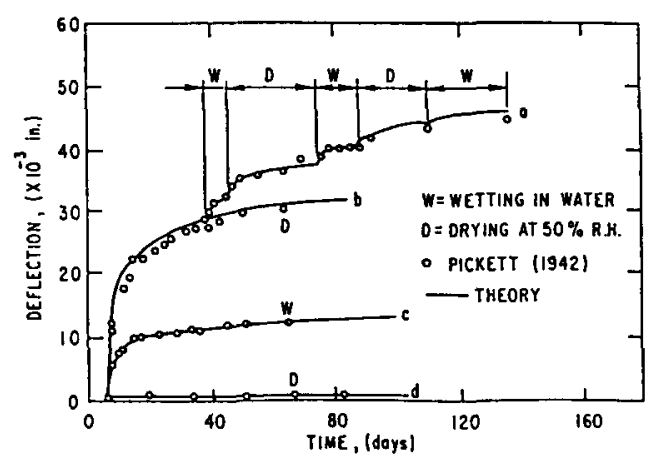

FIG. 14. Fits of Test Data by Pickett (27)

term must involve sign $\dot{h}$. Satisfactory fits of many further test data, which included drying but not wetting, were demonstrated in the preceding study (10).

\section{Conclusions}

1. The previous Bažant and Chern's theoretical conclusion that the physical mechanism of the additional creep caused by a temperature change, either heating or cooling (i.e., the transitional thermal creep or stress-induced thermal expansion) is the same as the physical mechanism of the additional creep due to humidity change, either drying or wetting (drying creep, Pickett effect, or the stress-induced shrinkage or swelling) is corroborated by comparisons with the existing test data.

2. The available test data confirm Bažant's hypothesis (7) that the 2. The avarent creep viscosity $\eta$ is a function of the variable $|\dot{H}|=\left|\dot{h}+a_{T} \dot{T}\right|$ (Eq. 6 ), rather than a general function of $\dot{h}$ and $\dot{T}$, and that consequently the 
stress in the stress-induced shrinkage and thermal expansion terms (Eqs. 10 and 11) ought to be multiplied by sign $\dot{H}$.

3. The physical mechanism of the apparent additional creep caused by changes of humidity as well as temperature may be explained principally by two effects: (1) The stress-induced shrinkage (or swelling) and the stress-induced expansion (or contraction), both being merely different manifestations of the same phenomenon; and (2) distributed tensile cracking (or strain-softening) of concrete, which represents an unknown portion of measured deformation, thus causing the true material shrinkage or swelling as well as thermal expansion to not be observable on load-free companion specimens. In addition to these two principal effects, further appreciable contributions arise from the irreversibility of unloading (i.e., resistance to contraction) after tensile cracking, and from the aging of concrete, i.e., the increase of its stiffness with age, unloading stiffness included (see Ref. 10).

\section{ACKNOWLEDGMENTS}

The work was sponsored by the U.S. Dept. of Energy and was performed in the Computational Mechanics Section (J. M. Kennedy, manager) of the Argonne National Laboratory, to which Z. P. Bažant served as consultant. Drs. J. M. Kennedy and A. H. Marchertas are thanked for their valuable assistance in the preparation of this paper.

\section{Appendix I. Test Data and Parameters Used}

Hansen and Eriksson (22). Cement mortar beams $(0.8 \times 2 \times 16$ in. $)$ were loaded to produce uniform bending moment between two supports ( $C=$ $58 ; s=0.3 ; E_{s}=6 \times 10^{6} ; \phi_{1}=6 ; n=0.174 ; m=0.306 ; \alpha=0.069 ; f_{c}^{\prime}=$ 6,500 psi; and $r=6$ ).

Pickett (27). Beams of cross section $2 \times 2$ in. and 32-in. span loaded by own weight plus a 50 -lb concentrated load at midspan $\left(E_{S}=1.58 \times 10^{8} ; c\right.$ $=55 ; s=0.3$; and $r=3.0$; other information: see Ref. 1).

Wallo, et al. (23). U-shaped specimens for flexural creep test had arms with cross-sectional dimensions $1 \times 1$ in. for a length of 9.375 in. ( $c=58$; $s=013 ; r=4 ; f_{c}^{\prime}=4,775 \mathrm{psi} ; t_{0}=60$ days $E_{s}=6 \times 10^{6} ; \phi_{1}=3.5 ; n=$ $0.134 ; m=0.31$, and $\alpha=0.045$ )

Fahmi, et al. (24). Hollow cylinders of 6-in. external diameter, 1 in.-wall thickness, and 40-in. length; 21-day compressive strength 5,840 psi; $E_{s}=$ $6 \times 10^{7} ; \phi_{1}=3.4 ; n=0.128 ; m=0.345 ; \alpha=0.04 ; \rho=2.4 ; r=5.0 ; U_{a}$ $=4,200 ; U_{h}=2,700 ; t^{\prime}=21$ days; $f_{c}^{\prime}=5,840 \mathrm{psi} ; h_{e}=0.5 ;$ permeability coefficient $a_{0}=5 \times 10^{-13} \mathrm{~m} / \mathrm{s} ;$ and thermal conductivity $=1.79 \mathrm{~J} / \mathrm{m}-\mathrm{sec}-{ }^{\circ} \mathrm{C}$.

Schneider (25). Solid cylinder with 3.15-in. diameter and 12-in. length; age at loading $=450$ days; $E_{s}=4.8 \times 10^{6} ; \phi_{1}=3.5 ; m=0.297 ; n=0.122$; $\alpha=0.05 ; \rho=0.8 ; r=1.6 ; U_{a}=4,200 ; U_{h}=2,700 ; f_{c}^{\prime}=6,890 \mathrm{psi} ; f_{t}^{\prime}=500$ psi; $\alpha=0.0007 ; a_{0}=5 \times 10^{-12} \mathrm{~m} / \mathrm{s} ;$ and thermal conductivity $=1.79$ $\mathrm{J} / \mathrm{m}-\mathrm{sec}-{ }^{\circ} \mathrm{C}$.

Anderberg and Thelandersson (15). Specimens made with a concentrical hole of diameter 4 in. to facilitate the deformation measurements $\left(E_{s}=4.8\right.$ $\times 10^{7} ; \phi_{1}=1.3 ; n=0.128 ; m=0.297 ; \alpha=0.042 ; U_{h}=2,700 ; U_{a}=4500 ;$ $\rho=1.6 ; r=2.3 ; a_{0}=1 \times 10^{-12} \mathrm{~m} / \mathrm{s}$, and thermal conductivity at $25^{\circ} \mathrm{C}=$ $\left.1.79 \mathrm{~J} / \mathrm{m}-\mathrm{sec}^{\circ} \mathrm{C}\right)$.

Hollington (21). Two large-size beams with reinforcing bars were tested. The beams were subjected to a uniform superimposed load of $56 \mathrm{lb} / \mathrm{ft}^{2}$; self-weight, and seasonal temperature changes $\left(c=58 ; s=0.3 ; \alpha_{0}=1.2\right.$ $\times 10^{-5} ; E_{s}=1 \times 10^{8} ; r=2.0 ; \rho=6.0$ ).

\section{Appendix II. References}

1. Anderberg, Y., and Thelandersson, S. (1976). "Stress and deformation characteristic of concrete at high temperatures, 2. Experimental investigation and material behavior model." Bulletin 54, Lund Inst. of Tech., Lund, Sweden.

2. Bažant, Z. P. (1970). "Constitutive equation for concrete creep and shrinkage based on thermodynamics of multiphase systems." Materials and Structures, 3, RILEM, Paris, France, 3-36; see also Rep. 68/1, Dept. of Civ. Eng., Univ. of Toronto, Toronto, Canada, 1968.

3. Bažant, Z. P. (1972). "Thermodynamics of interacting continua with surfaces and creep analysis of concrete structures." Nuclear Engrg. and Design, 20, 477-505; see also Cem. Concr. Res., 2(2), 1972, 1-16.

4. Bazant, Z. P. (1975). "Theory of creep and shrinkage in concrete structures: a precis of recent developments." Mechanics today, Vol. 2, S. NematNasser, ed., John Wiley, New York, N.Y.

5. Bažant, Z. P., and Thonguthai, W. (1978). 'Pore pressure and drying of concrete at high temperature." J. Eng. Mech. Div., ASCE, 104 (EMS), 1059-1079.

6. Bažant, Z. P. (1982). "Mathematical models for creep and shrinkage of concrete." Creep and shrinkage in concrete structures, Z. P. Bazant, and F. H. Wittmann, eds., John Wiley and Sons, New York, N.Y., 163-256.

7. Bažant, Z. P. (1984). Private communication in a letter to J.C. Chern and A. Marchertas.

8. Bažant, Z. P., Chern, J. C., and Thonguthai, W. (1981). "Finite element program for moisture and heat transfer in heated concrete." Nuclear Engrg.
Des., 68, 61-70.

9. Bažant, Z. P., Chern, J. C., and Thonguthai, W. (1981). TEMPOR2-User's manual. Dept. of Civ. Engrg., Northwestern Univ., Evanston, Ill

10. Bažant, Z. P., and Chern, J. C. (1985). "Concrete creep at variable humidity." Materials and Structures, RILEM, 18(103), 1-20.

11. Bažant, Z. P., and Chern, J. C. (1985). "Strain-softening with creep and exponential algorithm." J. of Engng. Mech., ASCE, 111(3), 391-415.

12. Bažant, Z. P., and Wu, S. T. (1974). "Creep and shrinkage law for concrete at variable humidity."J. Engrg. Mech., ASCE, 100 (EMG) 1183-1209.

13. Cilosani, Z. N. (1964). "On the true mechanism of creep of concrete." Beton i Zhelezobeton, (Moscow), 10, 75-78, (in Russian).

14. Fahmi, H. M., Polivka, M., and Bresler, B. (1972). "Effect of sustained and cyclic elevated temperature on creep of concrete." Cem. Concr. Res., 2 , $591-606$

15. Furamura, F. (1970). "Stress-strain relationship in compression of concrete at high temperatures." Trans. Architect. Inst., 174, Toyko, Japan.

16. Hansen, T. C., and Eriksson, L. (1966). "Temperature change effect on behavior of cement paste, mortar and concrete under load." $A C l \mathrm{~J} ., 63(4)$, 489-504.

17. Hollington, M. R. (1970). "A series of long-term tests to investigate the deflection of representative precase concrete floor component." Tech. Rep. No. TRA 442, Cement and Concrete Assoc., London, England.

18. Illston, J. M., and Sanders, P. D. (1973). "The effect of temperature change 
upon the creep of mortar under torsional loading." Mag. of Concrete Res., 25(84), 136-144

19. Pickett, G. (1942). "The effect of change in moisture content on the creep of concrete under a sustained load." J. Am. Conc. Inst., 38, 333-355; see also "Shrinkage stresses in concrete." J. Amer. Concr. Inst., 47, 1946, 165-204, 361-397.

20. Pfeiffer, P. A., Marchertas, A. H., and Bazant, Z. P. (1985). "Viscoelastic creep of high-temperature concrete." Proc. of 8th Int. Conf. on Siruct. Mech. in Reactor Tech., Brussels, Belgium, paper $\mathrm{H} 5 / 2$.

21. Purkiss, J. A., and Dougill, J. W. (1973). "Apparatus for compression tests on concrete at high temperatures." Mag. of Concrete Res., 25(83), 102-108.

22. RILEM Committee TC69. (1986). "State-of-art report on creep and shrinkage of concrete: mathema f modeling. "Preprints, 4th RILEM Int. Symp. on Creep and Shrinkage of Concrete: Mathematical Modeling," Z. P. Bazant, ed., held at Northwestern Univ., Evanston, Ill., 39-456.

23. Ruetz, W. (1968). "A hypothesis for the creep of hardened cement paste and the influence of simultaneous shrinkage." Proc. Int. Conf. Structure of Concrete, held in London, England, 1965, Cement and Concrete Assoc., London, England, 365-387; see also Deutscher Ausschuss fur Stahlbeton, Heft 183, 1966.

24. Schneider, U. (1976). "Behavior of concrete under thermal steady state and non-steady state conditions." Fire and Structures, 1, 103-115.

25. Schneider, U. (1982). "Behavior of concrete at high temperatures." Deutscher Ausschuss für Stahlbeton, Heft, 337, Berlin, West Germany.

26. Thelandersson, S. (1983). "On the multiaxial behaviour of concrete exposed to high temperature." Trans., 7th Int. Conf. on Struct. Mech. in Reactor Tech. Vol. $\mathrm{H}$, Paper H3/1.

27. Thelandersson, S. (1983). "On the multiaxial behavior of concrete exposed to high temperature." Nuclear Engrg. and Des., 75, 271-282.

28. Wallo, E. M., Yuan, R. L., Lott, J. L., and Kesler, C. E. (1965). "Sixth progress report: prediction of creep in structural concrete from short time tests." T\&AM Rep. No. 658, Univ. of Illinois, Urbana, Ill.

29. Wittmann, F. H. (1968). "Surface tension, shrinkage and strength of hardened cement paste." Materials and Structures, RILEM, 1, 547-552.

30. Wittmann, F. H. (1970). "Einfluss des Feuchtigkeitsgehaltes auf das Kriechen des Zementsteins." Rheol. Acta., 9, 282-287 (in German).

31. Wittmann, F. H. (1982). "Creep and shrinkage mechanisms." Creep and shrinkage in concrete structures, Z. P. Bazant and F. H. Wittmann, eds., John Wiley \& Sons, London, England, 129-161.

32. Wittmann, F. H., and Roelfstra, P. E. (1980). "Total deformation of loaded drying concrete." Cement and Concrete Res., 10,601-610. 